magnetic resonant mode below $T_{\mathrm{c}}$. Constant-energy scans show the mode at an energy of $47 \mathrm{meV}$, somewhat larger than the previously reported $40-\mathrm{meV}$ mode energies in the bilayer compounds.

JENNIFER BURRIS

\section{STM Maps Granular Structure of Superconductivity at Nanoscale Level}

Researchers at Lawrence Berkeley National Laboratory and the University of California, Berkeley, have used scanning tunneling microscopy (STM) to make nanometer-scale maps of "granular" superconductivity in the high-temperature superconductor $\mathrm{Bi}-2212\left(\mathrm{Bi}_{2} \mathrm{Sr}_{2} \mathrm{CaCu}_{2} \mathrm{O}_{8+\delta}\right)$. They verified their discovery with a second use of STM, employing individual nickel atoms as dopant probes to distinguish superconducting from nonsuperconducting regions in Ni-doped Bi-2212. J.C. Séamus Davis, a researcher with LBNL's
Materials Sciences Division and a professor of physics at UC-Berkeley, and his colleagues have shown that a high- $T_{c}$ superconductor with what is believed to be an essentially perfect crystal structure can exhibit granular superconductivity, (regions of superconductivity spatially separated from one another).

As reported in the January 24 issue of Nature, Davis's group cleaved perfect single crystals of B-2212, which split cleanly along the bismuth-oxygen plane lying immediately over the copper-oxygen plane. Using STM, the researchers were able to image individual atoms in the plane. In ultrahigh vacuum at very low temperature, the electronic states of the underlying copper-oxygen plane could also be sensed.

As the probe tip scanned over the plane, it measured differences in the current reaching the tip. Two kinds of regions of different conductance were revealed: $\alpha$ regions exhibiting relatively small energy gaps, typical of superconductivity; and $\beta$ regions with larger gaps.

From these spectral scans, "gapmaps" were constructed, showing that in the underdoped crystal, the $\alpha$ regions were roughly circular areas $<3 \mathrm{~nm}$ across, separated from one another and surrounded by narrow $\beta$ regions $\sim 2 \mathrm{~nm}$ wide. The researchers reported, however, that in "asgrown" slightly overdoped crystals, this nanoscale segregation was not evident.

Nickel atoms introduced into the copperoxygen planes of the $\mathrm{Ni}$-doped $\mathrm{Bi}-2212$ stood out because of the orientation of their surrounding clouds of charge: Cross shapes revealed the density of negative charges (electrons), and $x$ shapes showed that of positive charges (holes).

The Davis team surveyed B-2212 crystals a second time with the STM, looking for the cross-shaped and $x$-shaped resonances that were signatures of the individual nick-

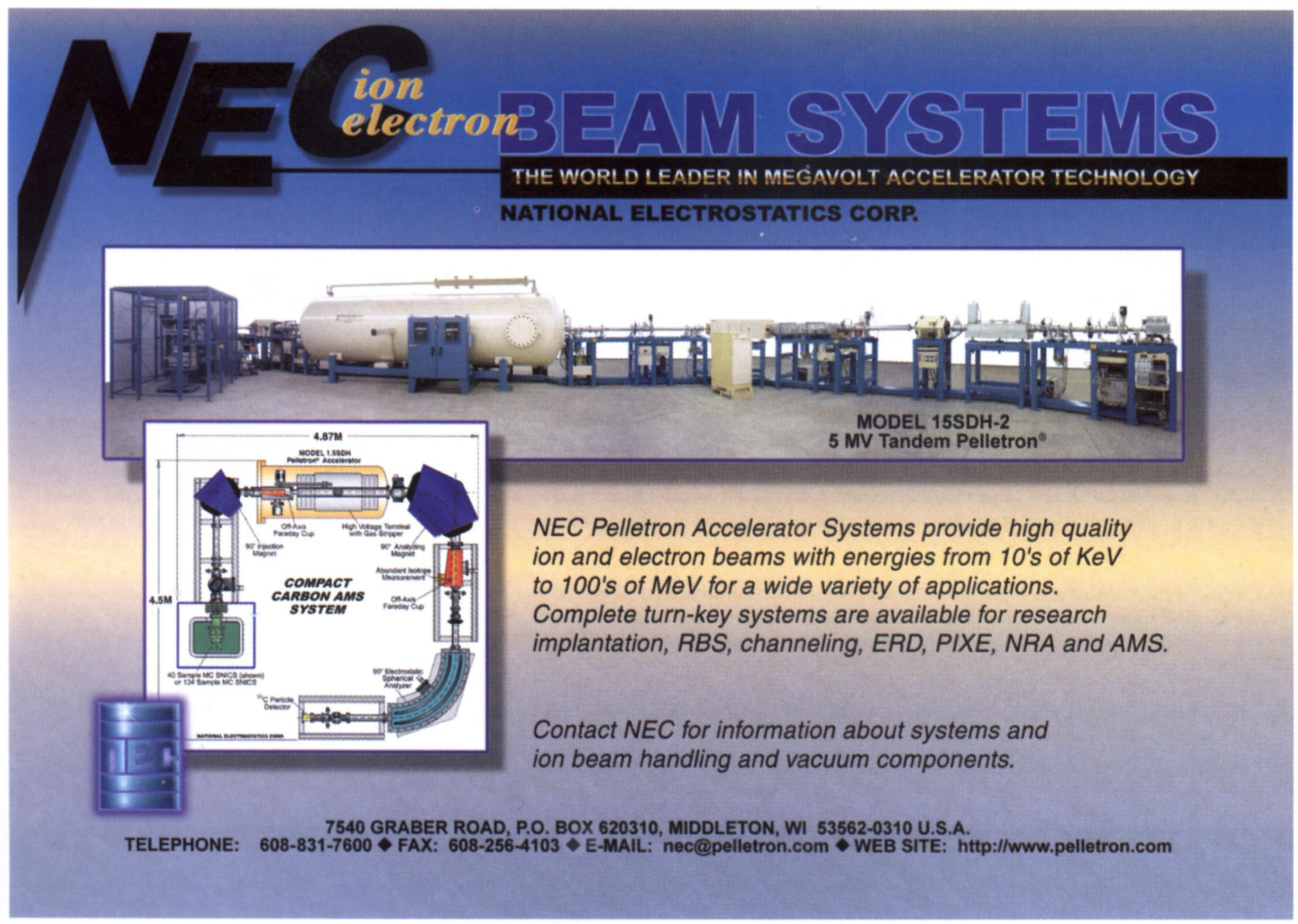

Circle No. 21 on Inside Back Cover 
el atoms they had introduced into the sample. In the underdoped compound, they found nickel impurities centered in $\alpha$ regions, but found none in $\beta$ regions.

Davis said, "A likely explanation is that nickel atoms are indeed present in other regions. But because these are not superconducting, there is no symmetrical particle-hole scattering to reveal the nickel impurities."

Taken together, the two STM techniques-high-resolution spectral surveys and the use of impurity resonances as local markers of superconductivity-not only show that superconductivity is segregated into discrete domains in underdoped B-2212, but also strongly suggest that this material displays granular superconductivity due to frustrated electronic phase separation, according to the research team.

"Since the domains are so close together," Davis said, "quantum-mechanical Josephson tunneling across the nonsuperconducting regions that separate them is probably what supports the long-range superconducting properties of this material."

\section{Protein Nanoarrays Developed for Studying Biological Processes}

Scientists at Northwestern University have utilized the method of dip-pen nanolithography to make arrays of proteins with features more than 1000 times smaller than those used in conventional arrays. This leads to nanoarrays with more than a million times the density of current commercial microarrays. Genetic and proteomic screening with so-called gene chips and proteomic arrays are allowing researchers to peer into the genetic code of individuals and develop leads for important therapeutic agents in the pharmaceutical industry. Current technologies use arrays of either proteins or DNA on the micrometer level as screening tools for drug testing and analyzing DNA, protein-protein interactions, and cell biology. Miniaturizing these arrays could dramatically improve their capabilities.

Led by Chad A. Mirkin, director of Northwestern's Institute for Nanotechnology, the research team combined expertise with Milan Mrksich of the University of Chicago and his group and also showed that the novel arrays could be used to study biological processes such as cell adhesion. This involves discovering and then writing a pattern of proteins that attracts a particular molecule.

As reported in the March 1 issue of Science (February 7 issue of the Science Express Web site, http://www.sciencexpress.org),
Mirkin's method of dip-pen nanolithography allowed the researchers to use an atomic force microscope tip as a nanopen to write out a tiny protein-dot array on a gold surface, dots as small as $100 \mathrm{~nm}$ in diameter. The gold surface between the dots was processed to prevent it from absorbing target proteins and disturbing the readings. When an array on a chip was exposed to protein targets in solution, the protein on the substrate (16-mercaptohexadecanoic acid, or MHA) bound its complementary proteins (lysozyme and rabbit immunoglobin). The atomic force microscope then read the chip and recorded a match where a change in height was detected.

\section{Three-Dimensional Waveguides Fabricated in Self-Assembled Photonic Crystals}

Scientists at the University of Illinois at Urbana-Champaign have used multiphoton polymerization and a laser scanning confocal microscope to create threedimensional waveguides or optical cavities inside self-assembled photonic crystals. Most photonic crystals with embedded features are built layer by layer, using conventional fabrication techniques.

Paul Braun, a researcher at the University's Frederick Seitz Materials Research Laboratory and the Beckman Institute for Advanced Science and Technology, said, "Colloidal self-assembly is an easier and cheaper alternative, especially for the generation of large-area photonic crystals."

As reported in the February 19 issue of Advanced Materials, to fabricate features, Braun and his colleagues-postdoctoral scientist Wonmok Lee and graduate student Stephanie Pruzinsky-assembled a colloidal crystal of uniform silica spheres $1.58 \mu \mathrm{m}$ in diameter. After removing the solvent, the researchers added a photoactive monomer to fill the spaces between the spheres. They then shined laser light through a microscope and into the crystal, polymerizing the monomer only at the microscope's focal point. The multiphoton absorption is only significant in a small volume $\left(V \sim \lambda^{3}\right)$ near the focal point. They removed the unpolymerized liquid, leaving the desired shape supported by the colloidal crystal matrix.

"By carefully moving the microscope's focal point through the crystal, complex three-dimensional patterns can be created," Braun said. "Unlike layer-by-layer techniques, we can move through the crystal in any direction, because the patterning is not a planar process."

The polymerization process creates a region with a different refractive index that can transmit light like an optical fiber.
"If you bend a fiber-optic cable too far, the light leaks out," Braun said. "With waveguides generated in photonicbandgap materials, you can bend light around much sharper corners."

\section{Artificial Intelligence Aids \\ Combinatorial Chemistry}

Chemical engineers at Purdue University have developed an automated system that uses combinatorial chemistry, a technique in which equipment systematically creates and tests thousands of chemical samples at the same time, that is, in parallel. According to the research team, this method can be used for many types of materials research.

As reported on January 25 at the 4th Annual International Symposium on Combinatorial Approaches for $\mathrm{New}$ Materials Discovery in San Diego, Calif., thousands of plastic beads were coated with different catalysts. All of the beads, each bearing its own individual catalyst, were tested simultaneously in the same experiment. The system then used a recently declassified infrared-sensor technology to screen each sample to evaluate its performance.

Associate professor of chemical engineering Jochen Lauterbach said, "We have the capability of making a hundred times more catalysts and screening them in the same amount of time that researchers previously needed to study one catalyst."

According to the researchers, a small percentage of the catalysts that are created are effective. Information is collected from both the best catalysts and the failed catalysts and fed into software that mimics the logical and intuitive thought processes of chemists. Even though the majority of the catalysts are not effective, the software uses the wealth of information gained from those failures to come up with entirely new catalysts.

Central to the method are two types of artificial-intelligence software: hybrid neural networks and genetic algorithms. The different types of software work together in a repeating two-phase cycle of discovery. First, hybrid neural networks analyze the formulas of the numerous catalysts or other materials created by the parallel technique. The neural networks determine the properties of the materials based on their chemical structures. In the second phase, genetic algorithms cull the best materials and eliminate the poor performers. The algorithms also generate "mutations" of the best materials to create better versions, and the software determines the chemical structures of those mutations.

The resulting formulas are returned to 